\title{
A histological analysis of granulomatous hepatitis
}

\author{
M. HUGHES AND H. FOX \\ From the Department of Pathology, University of Manchester
}

SYNOPSIS A study has been made of 33 cases of granulomatous hepatitis. Of these, 12 were eventually established as cases of sarcoidosis, nine as cases of tuberculosis, and four as examples of primary biliary cirrhosis. No clinical diagnosis was ever reached in six cases.

Analysis of the histological features of these cases shows that the presence of caseation or of tubercle bacilli will only allow for the diagnosis of approximately $50 \%$ of cases of tuberculosis. Histological features which are suggestive of tuberculosis are a paucity of granulomata, a scantiness of giant cells, a relatively mild surrounding chronic inflammatory cell infiltrate, and an absence of reticulin. The granulomata are more likely to be due to sarcoidosis if they are numerous, contain many giant cells, are confined to the portal areas, and show a well marked surrounding non-specific chronic inflammatory cell infiltrate.

Histological features of cases in which no clinical diagnosis was reached suggest that they are more likely to be due to sarcoidosis than to tuberculosis.

When interpreting liver biopsies the histological diagnosis of granulomatous hepatitis can usually be made without difficulty. Elucidation of the precise aetiology of such a hepatitis is, however, often difficult and sometimes impossible, and is, to a very considerable extent, dependent upon extensive clinical and laboratory investigation in which the histologist usually plays only a minor role. Many histopathologists when faced with the long list of possible causes of granulomatous hepatitis find themselves unable to detect any distinguishing features in such cases as to allow for the nomination of any specific one of these diseases as being the most likely. They are thus usually forced to the relatively unsatisfactory conclusion that the appearances are non-specific and compatible with tuberculosis, sarcoidosis, or other chronic granulomatous disease.

We have reviewed the histological and clinical features of all cases of granulomatous hepatitis seen at the Manchester Royal Infirmary during the years 1958-1971 in an attempt to determine whether or not careful histological study would reveal any features that would act as a guide towards a more specific diagnosis of hepatic granulomata.

\section{Material and Methods}

In the 13-year period covered by this study there were Received for publication 16 June 1972.
52 liver biopsies which were diagnosed as showing granulomatous hepatitis. An attempt was made to review all the sections from these cases together with the clinical records of the patients. Nineteen of these cases were, however, eventually excluded from this study-some because the clinical records were not available, others because the lesions present did not fulfil our diagnostic criteria of granulomatous hepatitis, and yet others from which the original slides had been lost and recutting of the block failed to reveal any granulomata.

Sections from each liver were studied without prior knowledge of the clinical diagnosis and were stained with haematoxylin and eosin, Gordon and Sweet's reticulin stain and a Ziehl-Neelson stain; a few sections were stained also with Masson's trichrome. Granulomata were only accepted as being present if they fulfilled the definition of Cameron (1962) as being 'a tumour-like mass of granulation tissue consisting of a focal vascularized aggregation of histiocytes and hypertrophied fibroblasts that assume a round shape to form epithelioid cells, occupy a central position and are surrounded by fibroblastic proliferation and usually a chronic inflammatory infiltrate of lymphocytes and plasma cells'. The site and number of the granulomata were noted as was also their reticulin pattern, the degree of the surrounding inflammatory cell infiltrate, and the absence or presence of caseation, other forms of necrosis, and giant cells. Other histological changes 
in the liver, such as inflammatory cell infiltration, bile stasis, liver cell necrosis, fibrosis or cirrhosis, were also sought for.

In the subsequent review of the clinical records fairly rigid criteria were used for the diagnostic grouping. Thus for a case to be accepted as being one of tuberculosis it was necessary for tubercle bacilli to have been identified within the granulomata or to have been isolated from elsewhere in the patient or for the patient to have made a good, and apparently specific, response to antituberculosis therapy. For a diagnosis of sarcoidosis to be sustained it was considered that a positive Kveim test should have been obtained and/or other typical manifestations of this disease, such as parotid involvement or hilar lymph node enlargement, should be present.

\section{Results}

During the period under review there were 1191 liver biopsies performed at this hospital; the incidence of reported granulomatous hepatitis was therefore $4.4 \%$ and, after exclusion of cases for the reasons stated above, the material we studied represented $2 \cdot 8 \%$ of the total liver biopsies.

The indications for liver biopsy and the presenting clinical features of these cases are detailed in Table 1 and it is clear that pyrexia, hepatomegaly, and weight loss were the outstanding features of this group of patients.

The final clinical diagnosis of these patients is shown in Table II and the histological features in their liver biopsies are detailed in Table III.

\section{Discussion}

There have been no previously reported large series of granulomatous hepatitis from this country but the incidence of granulomatous lesions in our series

\begin{tabular}{|c|c|}
\hline Clinical Feature & Number of Cases \\
\hline Pyrexia & 10 \\
\hline Pyrexia and hepatomegaly & 5 \\
\hline Hepatomegaly & 4 \\
\hline Hepatosplenomegaly & \\
\hline Weight loss & $\begin{array}{l}\text { ( } 3 \text { of these cases also had pyrexia) } \\
1 \\
\text { ( } 6 \text { other cases had associated } \\
\text { weight loss) }\end{array}$ \\
\hline Others ${ }^{1}$ & 6 \\
\hline Total & 33 \\
\hline
\end{tabular}

Table I Main clinical features of cases of granulomatous hepatitis

${ }^{1}$ Pulmonary symptoms, lymphadenopathy, parotid suelling. Apart from the cases of primary biliary cirrhosis, only one other case had jaundice as a clinical feature.

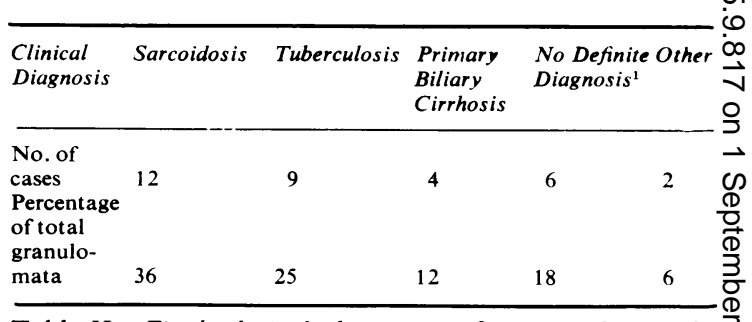

Table II Final clinical diagnosis of cases of granulomatous hepatitis

'One case of Hodgkin's disease and one of squamous cell carcinering of the bronchus.

of liver biopsies is similar to that reported in studies from North America (Wagoner, Anton, Gall, and $\frac{\varrho}{\varnothing}$ Schiff, 1953; Klatskin and Yesner, 1950): it is, however, much higher than that noted by Iverson, Christoffersen, and Poulsen (1970) in a report from Denmark. Too much stress should probably not be laid on differences in incidence which probably simply reflect variations in clinical recourse to liver용 biopsy.

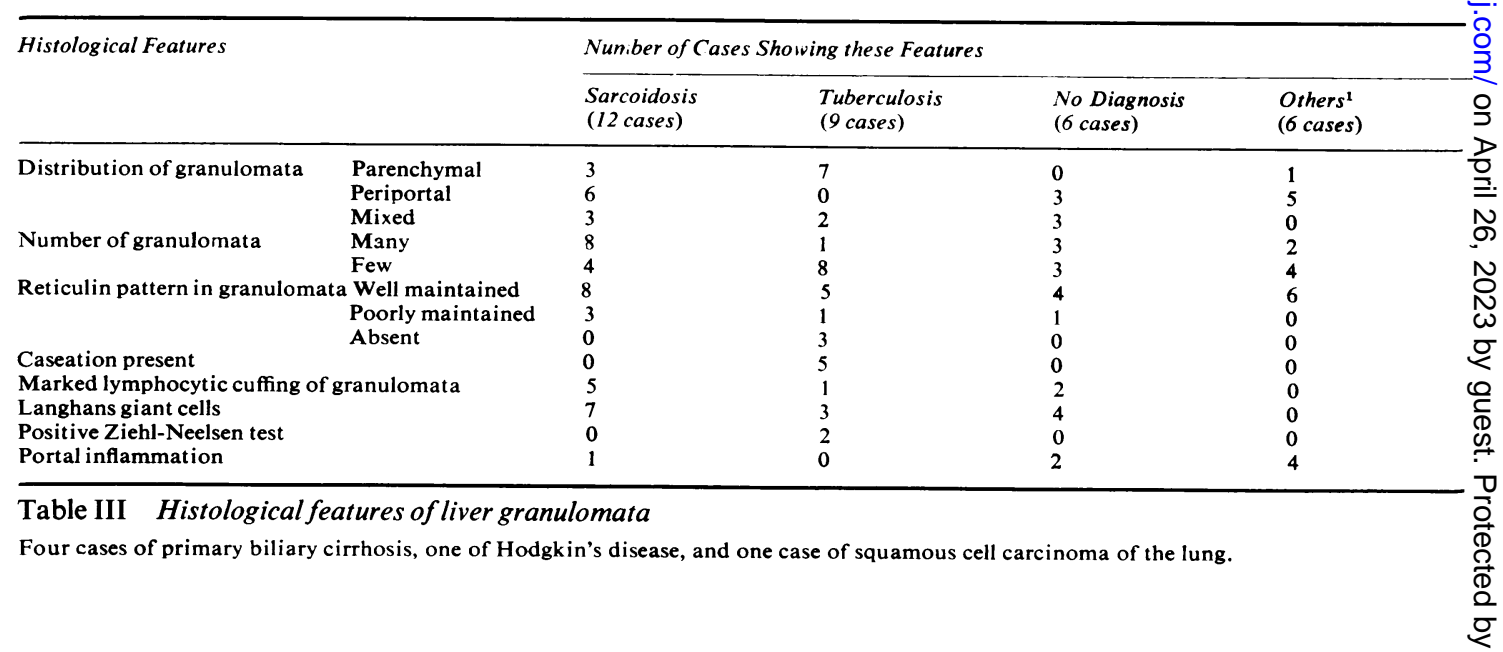


Our series corresponds with most others in that patients with tuberculosis or sarcoidosis make up the bulk of the cases in which an eventual clinical diagnosis was made. Such cases comprised $61 \%$ of our series and their combined incidence in other studies of granulomatous hepatitis has ranged from 33 to $66 \%$ (Guckian and Perry, 1966; Wagoner et al, 1953). The only other condition of any real importance as a cause of granulomatous hepatitis in this study was primary biliary cirrhosis and there was a noticeable absence of the myriad of other diseases that can be associated with hepatic granulomata. The absence of some diseases such as blastomycosis and histoplasmosis is presumably due to the very low incidence of these diseases in Great Britain as compared to that in the United States of America, whilst the lack of cases of infective mononucleosis and active viral hepatitis probably reflects the reluctance in this country to perform liver biopsies in such cases. No case of granulomatous hepatitis due either to $Q$ fever or brucellosis was seen despite the fact that during this period there were several liver biopsies from patients known to be suffering from these diseases. Cases in which no diagnosis was made represented $18 \%$ of our series and in other studies the incidence of such cases has ranged from 20 to $27 \%$.

Primary biliary cirrhosis is reasonably easy to separate out from the main bulk of patients with granulomatous hepatitis. The presence of lymphoid foci and destructive lesions of small bile ducts, together with the well marked inflammatory cell infiltrate, easily distinguishes this condition from either tuberculosis or sarcoidosis. The major problems facing the histopathologist are therefore those of differentiating granulomata due to sarcoidosis from those caused by tuberculosis and of attempting to resolve the possible aetiology of these cases in which no clinical diagnosis is made. A simple division of the granulomata can be made into those showing caseation and those without this change. Five cases of caseating granulomata were seen in this study and, although it has been maintained that caseation can occur in hepatic granulomata due to other conditions such as tularaemia, brucellosis, syphilis, mycotic infections, and Wegener's granuloma (Guckian and Perry, 1966), all of these were eventually established as cases of tuberculosis. This meant that four cases of tuberculosis did not show caseation and that therefore the absence of this change in no way rules out this diagnosis. The presence of tubercle bacilli in the granulomata is, of course, very strong evidence of tuberculosis but in only two out of nine cases of tuberculosis was a positive Ziehl-Neelson test obtained, this confirming Rubin's (1963) contention that tubercle bacilli are very rarely seen in liver biopsy material. It is clear therefore that the specific features of tuberculosis, namely, caseation and the presence of tubercle bacilli, are absent from approximately half of hepatic granulomata due to this disease and that any differentiation from sarcoidosis must often be made on other grounds. Of these the site of the granulomata would appear to be of some significance. In the 12 cases of sarcoidosis the granulomata showed a purely portal distribution in six, a purely parenchymal distribution in three, and a mixed parenchymal-portal pattern in three. In the nine cases of tuberculosis, however, the granulomata showed a purely parenchymal distribution in seven and a mixed parenchymal-portal distribution in two; in no case of tuberculosis were the granulomata situated solely in the portal regions. It would appear therefore that a purely portal distribution of hepatic granulomata is a strong indication of sarcoidosis but that the other patterns of distribution are of no distinguishing value. Two other possible distinguishing features between the two types of granuloinata are the presence of Langhans type giant cells and the degree of the perigranulomatous inflammatory cell infiltrate. Giant cells were seen in seven out of 12 cases of sarcoidosis but in only three of nine cases of tuberculosis whilst the surrounding inflammatory cell infiltrate was usually more marked in cases of sarcoidosis. A final histological point is that the granulomata tended to be more numerous in those cases due to sarcoidosis than in those caused by tuberculosis.

It has been claimed (Scheuer, 1968) that granulomata due to tuberculosis are poor in reticulin whilst those due to sarcoidosis contain abundant reticulin, but we have found the reticulin content of the granulomata to be of relatively little diagnostic value; thus the reticulin pattern of the granulomata was well maintained in eight of the 12 cases $(75 \%)$ of sarcoidosis and in five of the nine cases $(55 \%)$ of tuberculosis. On the other hand reticulin was totally absent from the granulomata of three cases of tuberculosis, a finding that was not present in any case of sarcoidosis.

Thus the diagnosis of sarcoidosis would appear most probable in cases in which the granulomata are non-caseating, numerous, contain many giant cells, are confined to the portal areas, and show a well marked surrounding inflammatory cell infiltrate. If, however, the granulomata are few, contain scanty giant cells, have only a mild surrounding cuff of chronic inflammatory cells, and contain no reticulin then tuberculosis would be the more likely diagnosis.

As in all studies of granulomatous hepatitis, a small proportion of cases defied clinical diagnosis. 
It has been suggested that all such patients have tuberculosis (Guckian and Perry, 1966) and should be given a course of antituberculosis therapy. Some patients certainly respond to this therapy but the possibility that they were suffering from transient and self-limiting diseases, possibly of viral origin, cannot be excluded. If the histological criteria we have outlined above are applied to the liver biopsies in this series from patients in whom no clinical diagnosis was made (cases 22-27) it would appear that the features are more suggestive of sarcoidosis than of tuberculosis. Thus of the six cases, three had numerous granulomata, three had a principally periportal distribution of the granulomata, four contained giant cells; in all the granulomata were non-caseating, and in none was reticulin absent from the granulomata. This is not to say that we consider all these cases to be definite examples of sarcoidosis but only that the histological findings suggest that these patients are more likely to fall into this diagnostic category than to be suffering from tuberculosis. It is of interest that in three of these cases a moderate number of eosinophils were seen in the granulomata; this can occur in sarcoidosis though it is not specific for this disease.

It has finally to be questioned whether there is a specific entity of 'idiopathic granulomatous hepatitis' (Fitzgerald, Fitzgerald, and Towers, 1971). At least one of the patients in our series has been known to have hepatic granulomata for over 10 yearso despite his remaining in good health and despite a亏 multiplicity of therapeutic régimes. The possibility that such a disease exists can neither be established or refuted from this series but we do feel that as careful study of the histological features of granulomatous hepatitis will enable the histopathologist tow give a more definite indication of the specifico aetiological disease process in a higher proportion of cases than is generally thought to be the case.

\section{References}

Cameron, R. (1962). Inflammation and repair. In Textbook of Pathology with Clinical Application, 2nd ed., edited by S. L N Robbins, p. 75. Saunders, Philadelphia and London.

Fitzgerald, M. X., Fitzgerald, O., and Towers, R. P. (1971). Granulomatous hepatitis of obscure aetiology. Quart. J. Med., 40,0 371-383.

Guckian, J. C., and Perry, J. E. (1966). Granulomatous hepatitis: an analysis of 63 cases and review of the literature. Ann. intern? Med., 65, 1081-1100.

Iversen, K., Christoffersen, P., and Poulsen, H. (1970). Epithelioid $\overrightarrow{-}$ cell granulomas in liver biopsies. Scand. J. Gastroent., Suppl $\mathscr{D}$ 61-67.

Klatskin, G., and Yesner, R. (1950). Hepatic manifestations of sar-D coidosis and other granulomatous diseases. Yale J. biol. Med.马 23, 207-248.

Rubin, E. (1963). Interpretation of the liver biopsy. Diagnostic criteria. Gastroenterology, 45, 400-412.

Scheuer, P. J. (1968). Liver biopsy interpretation, pp. 112-104. $\overrightarrow{0}$ Baillière, Tindall and Cassell, London.

Wagoner, G. P., Anton, A. T., Gall, E. A., and Schiff, L. (198) Needle biopsy of the liver VII. Experience with hepatic gra lomas. Gastrocinterology, 25, pp. 487-494. 